Proceedings of the International School and Conference on Optics and Optical Materials, ISCOM07, Belgrade, Serbia, September 3-7, 2007

\title{
Aperiodically Poled Nonlinear Crystals as Sources of Multi-Frequency Laser Radiation
}

\author{
A.A. Novikov* and G.D. Laptev \\ International Laser Center \\ M.V. Lomonosov Moscow State University, Russia
}

\begin{abstract}
The new multi-frequency process, which consists of three coupled nonlinear optical interactions: two parametric down-conversions and one up-conversion, in aperiodically poled nonlinear crystal is investigated. The spatial dynamics of wave intensities is studied in detail. The possibility of secondary simplification of coupled equations for correct describing the dynamics of wave interactions is demonstrated. The optimal conditions for parametrical instability of the initial stage of wave interactions are found.
\end{abstract}

PACS numbers: 42.25.-p, 42.15.Eq, 42.65.-k, 42.79.Nv

\section{Introduction}

Recently the quasi-phase matched (QPM) wave interactions are widely used in nonlinear optics due to the development of the fabrication technique of periodically poled nonlinear crystals (PPNCs). It is possible to simultaneously realize two coupled processes of wave interactions due to selection of modulation period of nonlinear coefficient in PPNCs [1]. In this case the reciprocal vector of nonlinear coefficient must compensate the phase mismatches of two processes at once. It should be noted that the compensation may be achieved only for limited numbers of optical frequencies [2]. The same difficulties occur at simultaneous implementation of more than two wave interactions.

The simultaneous realization of many nonlinear processes can be achieved in aperiodically poled nonlinear crystals (APNCs), in which nonlinear coupling coefficient changes according to aperiodic law (see, for example, [3-8]). It allows compensating phase mismatches of several nonlinear processes. However, the present-day APNCs in the same way as PPNCs also allow to simpler realize only

*corresponding author; e-mail: alexey_novikov@bk.ru 
one three-frequency process of wave interactions and have difficulties in implementation of several nonlinear processes due to dispersion of crystals.

In this paper we study a new type of multi-frequency process in APNC, which is designed by using new simple aperiodic law.

\section{The method of APNCs design for multi-frequency processes}

The idea of fabrication of APNCs is based on the generalization of modulation rule, which is used for fabrication of PPNC with modulation period $\Lambda$ :

$$
g(z)=\operatorname{sign}[\sin (2 \pi z / \Lambda)],
$$

where $g(z)$ is the modulation function of nonlinear coefficient of PPNC; $\Lambda=$ $2 \pi m / \Delta k ; \Delta k$ is the phase mismatch of wave vectors for three-frequency nonlinear process, which can be effectively realized in PPNC.

The generalization of Eq. (1) for the case of $N$ nonlinear process has the following form:

$$
g(z)=\operatorname{sign}\left[\sum_{j=1}^{N} a_{j} \sin \left(2 \pi z / \Lambda_{j}\right)\right],
$$

where $\Lambda_{j}=2 \pi m / k_{j} ; \Delta k_{j}$ is the phase mismatch of wave vectors for the $j$-th three-frequency process; $N$ is the number of simultaneous implemented nonlinear processes; $a_{j}$ is the numerical coefficient. Modulation function in Eq. (2) is

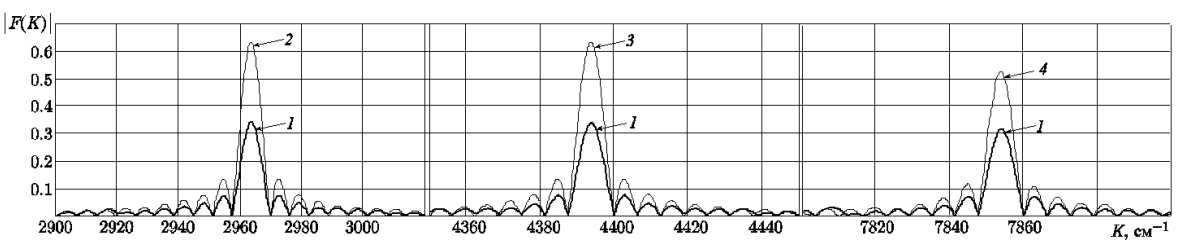

Fig. 1. Module of Fourier spectrum of function $g(z)$ of PPNC and APNC. $1-g(z)=$ $\operatorname{sign}\left[\sum_{j=1}^{3} \sin \left(2 \pi z / \Lambda_{j}\right)\right], 2-g(z)=\operatorname{sign}\left[\sin \left(2 \pi z / \Lambda_{1}\right)\right], 3-g(z)=\operatorname{sign}\left[\sin \left(2 \pi z / \Lambda_{2}\right)\right]$, $4-g(z)=\operatorname{sign}\left[\sin \left(2 \pi z / \Lambda_{3}\right)\right]$.

aperiodic in general case, and its Fourier spectrum has spectral components for compensating phase mismatches for several processes. For example, Fig. 1 illustrates the Fourier spectrum

$$
F(K)=\frac{1}{L} \int_{0}^{L} g(z) \exp (-\mathrm{i} K z) \mathrm{d} z
$$

of function $g(z)$ in APNC for implementation of three nonlinear processes $(N=3)$ in comparison with spectrum of function $g(z)$ in PPNC (see Eq. (1)). Here $\Lambda_{1}=21.2 \mu \mathrm{m}, \Lambda_{2}=14.3 \mu \mathrm{m}, \Lambda_{3}=8 \mu \mathrm{m}$, which are necessary for each of multi-frequency process (see below). One can see that spectral components in APNC, which are referring to different periods, are approximately equal to 0.3 , and the 
maximal absolute value of the Fourier spectral component for PPNCs is 0.63. Comparison of spectra of modulation functions in APNC and PPNC also shows that its spectral widths are approximately equal. This comparison is promising from the viewpoint of good efficiency of several simultaneous nonlinear processes in APNCs, which are created using the method under consideration.

\section{The dynamics of three coupled wave process with five waves}

We study the five-frequency process, which consists of simultaneous three nonlinear processes: (1) parametric down-conversion $\omega_{1} \rightarrow \omega_{2}+\omega_{3}$, (2) parametric up-conversion (sum-frequency generation) $\omega_{1}+\omega_{2} \rightarrow \omega_{4}$ and (3) parametric down-conversion $\omega_{5}=2 \omega_{1} \rightarrow \omega_{3}+\omega_{4}$. The five waves take part in this process, and two of their frequencies are divisible. It is well known that correlated photons with different frequencies are generating in non-degenerated parametric processes [9]. Thus the process under consideration is of interest for obtaining multipartite entanglement photon states [10].

For waves with wavelengths $\lambda_{1}=1.064 \mu \mathrm{m}, \lambda_{2}=2.129 \mu \mathrm{m}, \lambda_{3}=2.127 \mu \mathrm{m}$, $\lambda_{4}=0.709 \mu \mathrm{m}$, and $\lambda_{5}=0.532 \mu \mathrm{m}$ the five-frequency process can be implemented in APNC, in which $g(z)$ is defined by Eq. (2) at $\Lambda_{1}=21.2 \mu \mathrm{m}, \Lambda_{2}=14.3 \mu \mathrm{m}$, $\Lambda_{3}=8 \mu \mathrm{m}$. The process is described by the system of equations for amplitudes of interacting waves

$$
\begin{aligned}
& \mathrm{d} A_{1} / \mathrm{d} z=\mathrm{i} g(z)\left(\beta_{11} A_{2} A_{3} \mathrm{e}^{\mathrm{i} \Delta k_{1} z}+\beta_{21} A_{2}^{*} A_{4} \mathrm{e}^{-\mathrm{i} \Delta k_{2} z}\right), \\
& \mathrm{d} A_{2} / \mathrm{d} z=\mathrm{i} g(z)\left(\beta_{12} A_{1} A_{3}^{*} \mathrm{e}^{-\mathrm{i} \Delta k_{1} z}+\beta_{22} A_{4} A_{1}^{*} \mathrm{e}^{-\mathrm{i} \Delta k_{2} z}\right), \\
& \mathrm{d} A_{3} / \mathrm{d} z=\mathrm{i} g(z)\left(\beta_{13} A_{1} A_{2}^{*} \mathrm{e}^{-\mathrm{i} \Delta k_{1} z}+\beta_{33} A_{5} A_{4}^{*} \mathrm{e}^{-\mathrm{i} \Delta k_{3} z}\right), \\
& \mathrm{d} A_{4} / \mathrm{d} z=\mathrm{i} g(z)\left(\beta_{24} A_{1} A_{2} \mathrm{e}^{\mathrm{i} \Delta k_{2} z}+\beta_{34} A_{5} A_{3}^{*} \mathrm{e}^{-\mathrm{i} \Delta k_{3} z}\right), \\
& \mathrm{d} A_{5} / \mathrm{d} z=\mathrm{i} g(z) \beta_{35} A_{3} A_{4} \mathrm{e}^{\mathrm{i} \Delta k_{3} z},
\end{aligned}
$$

where $A_{j}$ is the amplitude of wave with frequency $\omega_{j}=2 \pi / \lambda_{j} ; \Delta k_{1}=k_{1}-k_{2}-k_{3}$, $\Delta k_{2}=k_{4}-k_{1}-k_{2}, \Delta k_{3}=k_{5}-k_{3}-k_{4}$ are the phase mismatches for processes (1), (2), and (3), respectively; $\Lambda_{j}=2 \pi m / \Delta k_{j} ; \beta_{l j}=4 \pi \omega_{j} d_{j}^{(l)} /\left(c n_{j}\right)$ is the nonlinear coupling coefficient; $n_{j}$ is the refractive index of crystal for wave at frequency $\omega_{j}$; $d_{j}^{(l)}$ is the effective nonlinear coefficient for wave at frequency $\omega_{j}$ in the process $l(l=1,2,3$, see above); $c$ is the velocity of light in vacuum. The following conditions are fulfilled for nonlinear coupling coefficients

$$
\beta_{11}=\beta_{12}+\beta_{13}, \quad \beta_{24}=\beta_{21}+\beta_{22}, \quad \beta_{35}=\beta_{33}+\beta_{34} .
$$

The system of Eqs. (4)-(8) is satisfying the condition

$$
\sum_{j=1}^{5} I_{j}(z)=\text { const }
$$

where $I_{j}(z)=\left|A_{j}(z)\right|^{2}$.

Figure 2 presents the results of numerical solutions of system (4)-(8) at $\beta_{11}=\beta_{24}=\beta_{35}, \beta_{12}=\beta_{13}, \beta_{21}=2 \beta_{22}, \beta_{34}=3 \beta_{33}, a_{1}=a_{2}=a_{3}$. The calculations have shown that the presence of intensity waves (pump) with frequencies $\omega_{1}$ 

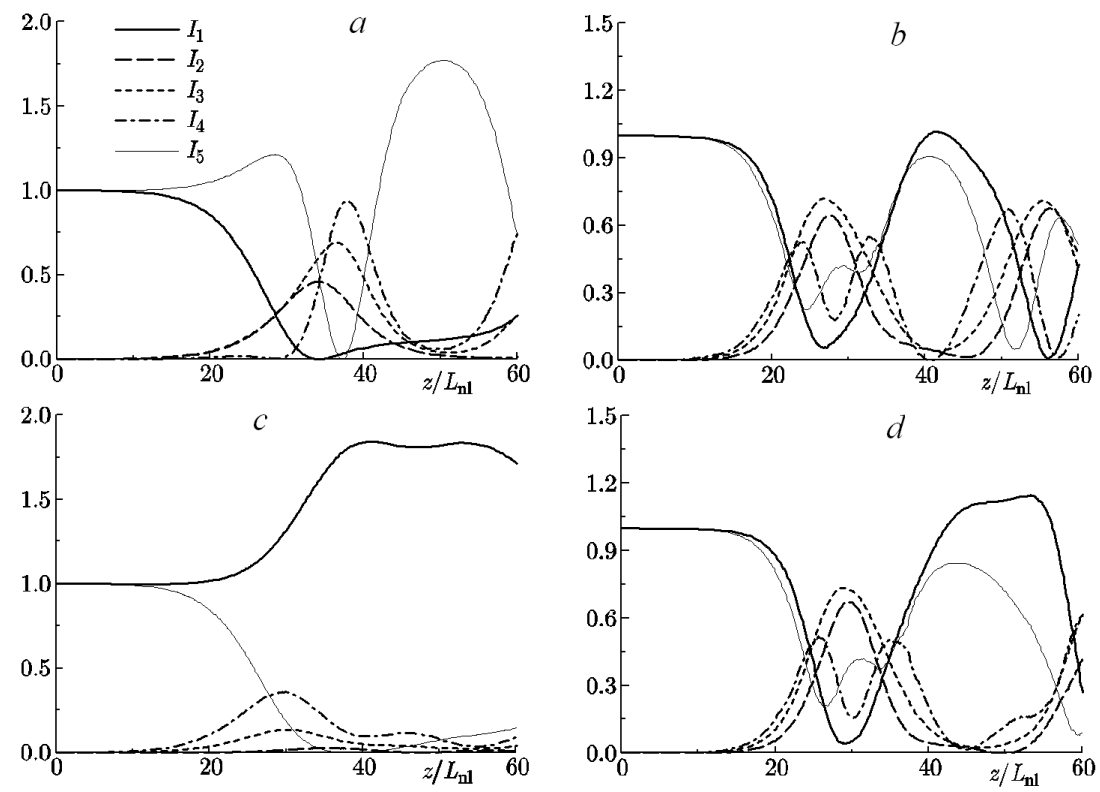

Fig. 2. Intensities $I_{j}$ (normalized on $I_{1}(0)$ ) of wave with frequency $\omega_{j}$ as functions of crystal length: (a) $\operatorname{Re} A_{1}(0)=\operatorname{Re} A_{5}(0), \operatorname{Re} A_{2,3,4}(0)=10^{-2} \operatorname{Re} A_{1}(0), \operatorname{Im} A_{j}(0)=0$; (b) $\operatorname{Re} A_{1}(0)=\operatorname{Im} A_{5}(0), \operatorname{Re} A_{2,3,4}(0)=10^{-2} \operatorname{Re} A_{1}(0), \operatorname{Im} A_{1,2,3,4}(0)=\operatorname{Re} A_{5}(0)=0$; (c) $\operatorname{Im} A_{1}(0)=\operatorname{Re} A_{5}(0), \operatorname{Re} A_{2,3,4}(0)=10^{-2} \operatorname{Im} A_{1}(0), \operatorname{Re} A_{1}(0)=\operatorname{Im} A_{2,3,4,5}(0)=0$; (d) $\operatorname{Im} A_{1}(0)=\operatorname{Im} A_{5}(0), \operatorname{Re} A_{2,3,4}(0)=10^{-2} \operatorname{Im} A_{1}(0), \operatorname{Re} A_{1}(0)=\operatorname{Im} A_{2,3,4}(0)=0$.

and $\omega_{5}$ is necessary for the process implementation. In calculations we supposed $I_{1}(0)=I_{5}(0), I_{2,3,4}(0) \ll I_{1,5}(0)$. Coordinate $z$ and intensities $I_{j}$ are normalized on $L_{\mathrm{nl}}=1 /\left[\beta_{11}\left(I_{1}(0)\right)^{1 / 2}\right]$ and $I_{1}(0)$, respectively.

Figure 2 shows that the effective energy exchange between interacting waves takes place in the process under consideration. The dynamics of energy exchange has the complex character, which can be changed in dependence on ratio between real and image parts of amplitudes of pump waves at the crystal input. Our analysis also shown that the effective energy exchange takes no place at $a_{2}>a_{1,3}$.

As a rule the condition $L_{\mathrm{nl}} \gg \max \left\{\Lambda_{1}, \Lambda_{2}, \Lambda_{3}\right\}$ is fulfilled for PPNCs. In this connection we have calculated the intensities of interacting waves using system (4)-(8), in which the fast changing multipliers $g(z) \exp \left(\mathrm{i} \Delta k_{j} z\right)$ and $g(z) \exp \left(-\mathrm{i} \Delta k_{j} z\right)$ were changed by its average values $q_{j}$ and $q_{j}^{*}$ respectively, where

$$
q_{j}=\frac{1}{L} \int_{0}^{L} g(z) \mathrm{e}^{\mathrm{i} \Delta k_{j} z} \mathrm{~d} z .
$$

Thus the system (4)-(8) has the form

$$
\mathrm{d} A_{1} / \mathrm{d} z=\mathrm{i}\left(q_{1} \beta_{11} A_{2} A_{3}+q_{2}^{*} \beta_{21} A_{2}^{*} A_{4}\right),
$$




$$
\begin{aligned}
& \mathrm{d} A_{2} / \mathrm{d} z=\mathrm{i}\left(q_{1}^{*} \beta_{12} A_{1} A_{3}^{*}+q_{2}^{*} \beta_{22} A_{4} A_{1}^{*}\right), \\
& \mathrm{d} A_{3} / \mathrm{d} z=\mathrm{i}\left(q_{1}^{*} \beta_{13} A_{1} A_{2}^{*}+q_{3}^{*} \beta_{33} A_{5} A_{4}^{*}\right), \\
& \mathrm{d} A_{4} / \mathrm{d} z=\mathrm{i}\left(q_{2} \beta_{24} A_{1} A_{2}+q_{3}^{*} \beta_{34} A_{5} A_{3}^{*}\right), \\
& \mathrm{d} A_{5} / \mathrm{d} z=\mathrm{i} q_{3} \beta_{35} A_{3} A_{4} .
\end{aligned}
$$

Our calculations have demonstrated that the solution of system (4)-(8) and system (12)-(16) are in a good agreement for $z \approx 50 L_{\mathrm{nl}}$. We note that such simplification of equations allows to essentially decrease the time of numerical solution of equations and develops the quantum theory of the process under consideration (see $[10])$.

The system (12)-(16) has the simple solution in the case of $\operatorname{Re}\left\{q_{2} q_{3} A_{1}^{2} A_{5}^{*}\right\}=$ 0 . For example, the solution for $A_{2}(z)$ is the following:

$$
A_{2}(z)=C_{1} \operatorname{ch}(\Gamma z)+C_{2} \operatorname{sh}(\Gamma z)+C_{3},
$$

where

$$
\Gamma^{2}=\left(q_{1}^{2} \beta_{12} \beta_{13}-\left|q_{2}\right|^{2} \beta_{22} \beta_{24}\right)\left|A_{1}(0)\right|^{2}+\left|q_{3}\right|^{2} \beta_{33} \beta_{34}\left|A_{5}(0)\right|^{2} ;
$$

$C_{j}$ is defined by initial condition. The parametric instability of initial state takes place at $\Gamma^{2}>0$. In this case the intensities of interacting waves are increased with crystal length. The dependences of Fig. 2 at small $z$ correspond to parametric instability regime.

\section{Conclusion}

The dynamics of new multi-frequency process, which can be realized in APNCs, was investigated. The multi-frequency processes in the considered APNCs are very promising for compact frequency converter and applications of quantum optics.

This research was partially supported by Russian Foundation for Basic Research (grant 05-02-17017), grant of President of Russian Federation (-4708.2006.2) and project INTAS (100008-7904).

\section{References}

[1] R.L. Byer, J. Nonlin. Opt. Phys. Mater. 6, 549 (1997).

[2] A.S. Chirkin, V.V. Volkov, G.D. Laptev, E.Yu. Morozov, Quantum Electron. 30, 847 (2000).

[3] J. Feng, Y.Y. Zhu, N.B. Ming, Phys. Rev. B 41, 5578 (1990).

[4] X. Liu, Z. Wang, J. Wu, D. Shen, N. Ming, Phys. Rev. B 58, 12782 (1998).

[5] Y.B. Chen, C. Zhang, Y.Y. Zhu, S.N. Zhu, H.T. Wang, N.B. Ming, Appl. Phys. Lett. 78, 577 (2001).

[6] X. Liu, Z. Wang, J. Wu, D. Shen, N. Ming, Phys. Rev. A 58, 4956 (1998).

[7] S.N. Zhu, Y.Y. Zhu, Y.Q. Qin, H.F. Wang, C.Z. Ge, N.B. Ming, Phys. Rev. Lett. 78, 2752 (1997).

[8] L.M. Zhao, B.Y. Gu, Y.S. Zhou, F.H. Wang, J. Appl. Phys. 94, 1882 (2003). 
[9] D.F. Walls, G.J. Milburn, Quantum Optics, Springer-Verlag, Berlin 1994.

[10] A.S. Chirkin, M.Yu. Saigin, Acta Phys. Hung. B 23, 63 (2006). 